\title{
METABOLIC EFFECTS OF ALLOPURINOL AND ALLO-XANTHINE
}

\author{
BY
}

\author{
R. W. RUNDLES
}

From the Haematology Laboratory, Duke Hospital, and the

Department of Medicine, Duke University School of Medicine, Durham, North Carolina, U.S.A.

An additional comment regarding the origin of allopurinol may now be appropriate. This new type of agent, which many investigators have found to be uniquely useful in the treatment of gout, secondary hyperuricaemia, and hyperuricosuria, is a product of the programme of study of antimetabolites relating to purines and pyrimidines undertaken by Hitchings and Elion (1954) more than 20 years ago. In the early 1950s, two thiopurine compounds of major importance were first synthesized in their laboratory: thioguanine (TG) and 6-mercaptopurine (6-MP). Biological tests showed that both compounds were effective as antipurines and anti-tumour and antileukaemic activities were soon demonstrated (Clarke, Philips, Sternberg, and Stock, 1954). During the following years, as the range of clinical usefulness of the thiopurines was explored, many investigators studied their mechanism of action. This turned out to be complicated, since many interrelated biochemical effects were produced. In the search for better agents, several hundred related compounds were synthesized and subjected to biological study. Thiopurines with different substituents on the sulphur atom proved to have variable and unpredictable effects in animal species and in man (Elion, Callahan, Hitchings, Rundles, and Laszlo, 1962). Comparative studies of drug metabolism were undertaken to explain these discrepancies (Elion, Callahan, Rundles, and Hitchings, 1963b; Hitchings, 1963). One particular finding relating to the degradation of 6-MP led to the present somewhat tangential line of investigation. When therapeutic doses of 6-MP were given to man, 5 to 7 per cent. of the free compound was ordinarily excreted in the urine within a few hours, while 25 to 30 per cent. of the compound was oxidized to the inert metabolite 6-thiouric acid (TU). The magnitude of this oxidative degradation, known to be mediated by the enzyme xanthine oxidase, led to the idea that an enzyme inhibitor might be a useful chemotherapeutic adjuvant (Elion, Callahan, Nathan, Bieber, Rundles, and Hitchings, 1963a).

The biochemical considerations, the enzymatic and experimental studies which led to the selection of 4-hydroxypyrazolo (3, 4-d) pyrimidine (4-HPP, $\vec{z}$ allopurinol) for detailed study have been presented by Dr. Hitchings and Miss Elion. The toxicity of the compound when given in ascending doses $t o$ mice, rats, and dogs was minimal, and in the spring of 1962 we began to study its effect in patient (Rundles, Wyngaarden, Hitchings, Elion, anī Silberman, 1963).

Case 1.-The first individual to whom we gave allopurinol was a 45-year-old school teacher, who was discovered to have chronic granulocytic leukaemia late in May, 1962 (Fig. 1). She was started on 6-MP, $200 \mathrm{mg}$. daily, and during the first 2 weeks of treatment there was little discernible effect on her leukaemic status. Studies of the metabolism of 6-MP given with and without allopurinol were carried out. When $150 \mathrm{mg}$. 6-MP was given orally alone, $7 \cdot 2$ per cent. of the compound was excreted in the urine as free 6-MP and $25 \cdot 5$ per cent. was converted to 6-TU; 2 days later $150 \mathrm{mg}$. 6-MP with $300 \mathrm{mg}$. allopurinol were given simultaneously, and on this occasion, $29 \cdot 2$ per cent. of the administered dose was excreted in the urine as free 6-MP and only 3.4 per cent. as TU.

During the following 6 weeks, 6-MP therapy was maintained and the patient's leukaemic status came under good control. Another study of 6-MP metabolism was carried out with the disease in remission, $150 \mathrm{mg}$. of the compound being given with $75 \mathrm{mg}$. allopurinol. A 4-fold $\mathrm{N}$ increase in the urinary excretion of free 6-MP was again observed with a reduction in 6-TU excretion to 10 per cent. (Fig. 1, overleaf).

To investigate possible changes in the metabolism of endogenous purines produced by the administration of allopurinol, the patient had been started on a constant purine, constant protein diet. When equilibrium $\mathscr{尺}$ occurred, the serum uric acid was around $3 \cdot 2 \mathrm{mg} . / 100 \mathrm{ml}$. 


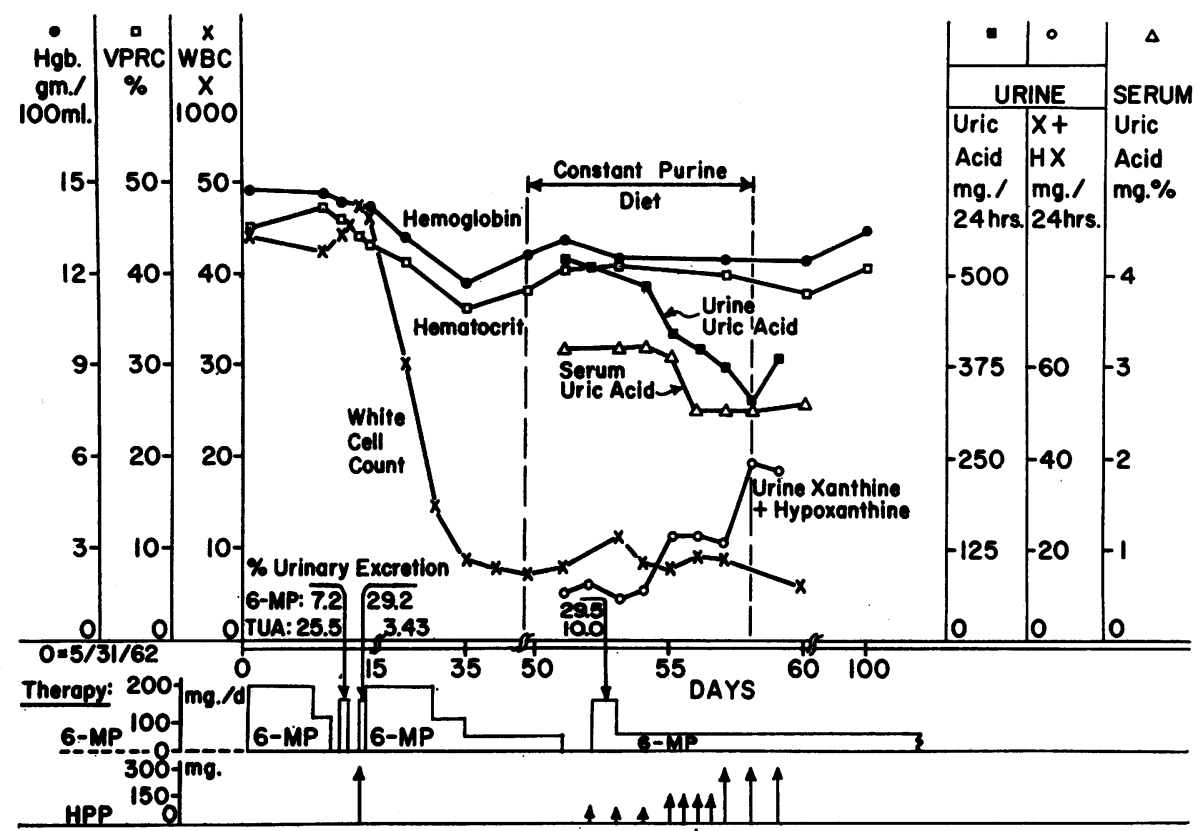

Fig. 1.-Effects of allopurinol therapy in a patient receiving 6-mercaptopurine.

and the urinary urate excretion around $500 \mathrm{mg} . / 24$ hours. As the dose of allopurinol was increased in the course of one week from 75 to 150 to $300 \mathrm{mg}$./day, the urinary uric acid excretion fell to $375 \mathrm{mg}$./day or below, and the serum uric acid to $2.5 \mathrm{mg} . / 100 \mathrm{ml}$. (Fig. 1). The excretion of xanthine plus hypoxanthine in the urine rose from about $10 \mathrm{mg}$. to about $\mathbf{4 0} \mathrm{mg}$. $/ 24$ hours. This provided the first biochemical evidence that the administration of allopurinol did in fact inhibit the activity of xanthine oxidase in vivo in man.

Finally, when the study had been completed and the patient was being examined preparatory to discharge from hospital, it was found that she had developed a lower abdominal tumour. It turned out that she was pregnant and that early symptoms of this had actually led to the discovery of granulocytic leukaemia. She was treated with 6-MP throughout the remainder of the pregnancy with no untoward developments. The pregnancy terminated with the birth of a normal boy who now, more than 3 years later, appears to be active, energetic, and entirely well.

Our subsequent work has been primarily concerned with verifying and extending these initial observations: determining the range of drug tolerance, the degree and regularity of its effect on 6-MP metabolism, and its effect on the metabolism of natural purines in a variety of diseases, and in searching for evidence of possible acute and/or chronic toxicity (Rundles and others, 1963).

Allopurinol turned out to be an exceptionally well-tolerated drug. We have ordinarily given it in two to three divided doses without regard to meak $\vec{\varphi}$ in total amounts up to 1 to $1 \cdot 2 \mathrm{~g}$./day. The minimas effective dose is 200 to $300 \mathrm{mg}$./day. The largest doses given have produced no definite evidences of toxicity, and the maximum tolerated dose has not yet been determined for man. When given in therapeutic amounts for a few weeks or months, $\mathbb{D}$ allopurinol has produced virtually no adverse haematological, renal, hepatic, gastrointestinal, peripheral, or central nervous system side-effects. Electro-encephalograms made when the serum uric acid level was reduced to as low as 2 to $3 \mathrm{mg}$. per $100 \mathrm{ml}$. were normal. A battery of tests designed to detect evidence of cumulative or chronic drug toxicity was repeated at intervals over periods of therapy extending up to 2 to 3 years. These disclosed no evidence of adverse haematological, hepatic, or renal effects. In patients with pre-existing bone marrow or renal disease, no additional impairment of function was produced by allopurinol therapy. There has been no evidence that other enzymatic functions of xanthine oxidase have been $N$ disturbed-no reduction in alcohol tolerance, no $\mathrm{N}$ impairment of iron absorption, storage, or incor- N poration into haemoglobin, etc. (Wyngaarden, Rundles, and Metz, 1965; Rundles, Metz, and Silberman, 1966b).

An unusual type of drug reaction did occur, however, in 2 to 3 per cent. of the first patients 
treated with allopurinol. The reaction was predominantly a pruritic, maculopapular skin eruption with fever, malaise, and aching. All signs and symptoms of toxicity subsided in a few days without residua after administration of the drug was stopped. In our patients, the reaction occurred particularly in those with seriously impaired renal function. The pathogenesis of this reaction is being studied further.

The most conspicuous metabolic effect of allopurinol is a reduction in the serum uric acid concentration which has been observed to occur in patients with acute and chronic leukaemia, multiple myeloma, myeloproliferative diseases, malignant lymphomata, psoriasis, renal disease of diverse aetiology, and primary and secondary gout. The effect was qualitatively the same in patients with haematopoietic malignancies in relapse and in remission, in non-malignant entities, and in normal subjects.

Case 2.-A 43-year-old man with previously untreated advanced multiple myeloma excreted 10 to $11 \cdot 5 \mathrm{~g}$. Bence Jones protein and about $540 \mathrm{mg}$. uric acid in the urine daily. His serum uric acid level was 5.6 to $6.0 \mathrm{mg}$. $/ 100$ $\mathrm{ml}$. He was given allopurinol in a dose of 400 to 600 mg./day (Fig. 2). The serum uric acid concentration and $\square$ the amount of urate excreted in the urine fell precipitously in parallel to about $3 \mathrm{mg} . / 100 \mathrm{ml}$. and $400 \mathrm{mg} . / 24 \vec{\Rightarrow}$ hours, respectively. There was no change, however, in $\stackrel{5}{+}$ the proteinuria or in the other primary manifestations of the plasma cell myeloma. The plan of therapy was then $\overline{ }$ changed to one using prednisone and L-phenylalanine mustard. On this regimen he had an unusually complete remission in his disease which has now been maintained for nearly 4 years.

The effect of allopurinol on the serum uric acid in twelve patients with gout is shown in Fig. 3 (overleaf).

Serum uric acid levels ordinarily began to fall within 2 to 3 days in patients given 300 to $600 \mathrm{mg}$. allopurinol daily, and the maximum suppression with a given dose was usually achieved in about 3 to 5 days. Abnormally high serum uric acid levels were reduced to normal or below in virtually all patients. The few exceptions were those who had extensive tophaceous deposits and/or poor renal function, and those who developed reactions to the drug. The serum uric acid level could generally be maintained more or less indefinitely at any desired level, as low as 2 to $3 \mathrm{mg}$. $/ 100 \mathrm{ml}$., by appropriate adjustments

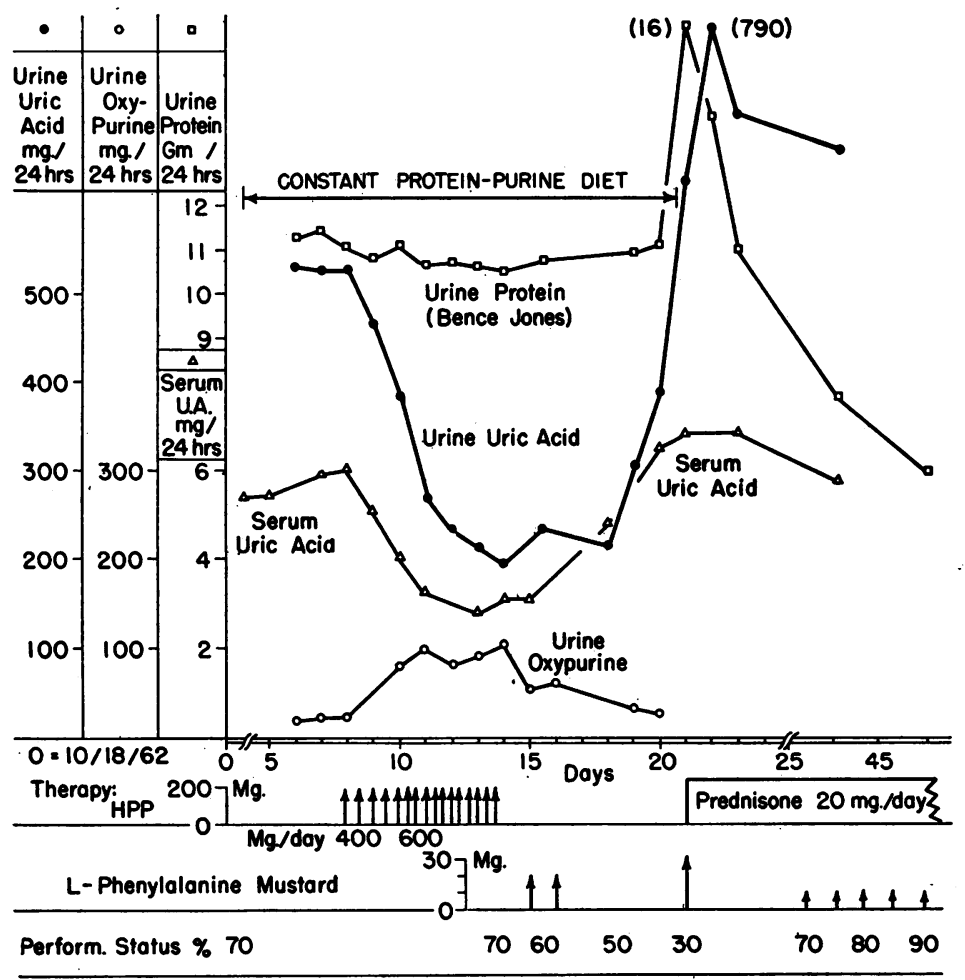

Fig. 2.-Effects of allopurinol therapy in a patient with untreated multiple myelomata. 


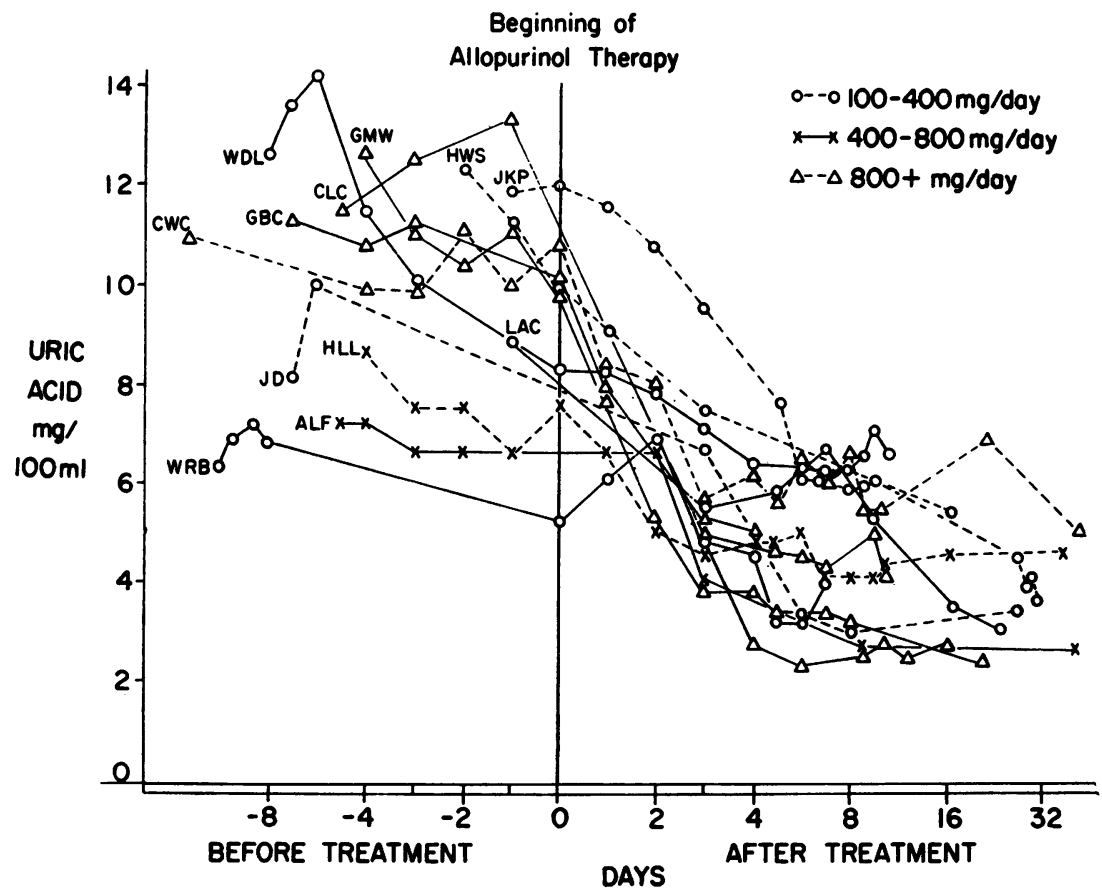

Fig. 3.-Effects of allopurinol therapy in twelve patients with gout.

in the dose of allopurinol. The fall in the serum uric acid level paralleled the reduction in urate excreted in the urine, particularly in patients with normal or increased uric acid production. On occasion, when the administration of allopurinol was suspended, both the serum uric acid concentration and the amount excreted in the urine increased together in parallel over a period of 5 to 7 days.

That the reduction in serum uric acid concentration and in urate excretion resulted from xanthine oxidase inhibition, producing a block in the conversion of hypoxanthine and xanthine to uric acid, was shown by the concomitant rise in the excretion of oxypurines during allopurinol therapy. By column and paper chromatography, xanthine was found to account for 55 to 75 per cent. of the total oxypurine increment and hypoxanthine for 25 to 45 per cent. There were only trivial changes in the excretion of other purine bases. The serum oxypurine levels in treated patients rarely exceeded $1 \mathrm{mg} . / 100$ $\mathrm{ml}$. and were usually in the range of 0.3 to $0.5 \mathrm{mg} . / 100$ $\mathrm{ml}$.- - only a little above the normal values of 0.1 to 0.3 mg./100 ml. (Rundles and others, 1963; Rundles and others, 1966b; Rundles, Elion, and Hitchings, 1966a). The finding by Goldfinger, Klinenberg, and Seegmiller (1965) that hypoxanthine and xanthine are cleared by the kidney at close to the glomerular filtration rate, in striking contrast to uric acid, explains why this should occur.

Our original expectation was that the reduction in urinary urate excretion produced by allopurinol would be reasonably well balanced by an increased excretion of hypoxanthine and xanthine, with total purine excretion remaining about constant. Findings to the contrary have been of considerable interest (Rundles and others, 1963). Although some variation has been found in individual patients, having different diseases studied at various intervals after the beginning of treatment, some with extensive tophi and/or seriously impaired renal function, etc.,을 the findings of all investigators are now in substantial agreement. The general trends may be illustrated by the findings in fourteen of our patients with gout who were studied with particular reference to total purine excretion before and after allopurinoltherapy.

All fourteen had been given allopurinol for 3 to $16 \frac{}{2}$ months. The rise in oxypurine excretion approximated or even exceeded the fall in uric acid excretion during the initial days or weeks of treatment in some instances, but $\$$ thereafter there was a gradual decrease in total purine excretion in the majority of individuals studied. Then magnitude of the deficit varied somewhat from patient to 9 patient, but it was fairly constant in a given individual.N The deficit tended to be greater in patients who had beenn overproducers of uric acid and in those who took larger $\omega$ doses of allopurinol for longer periods of time. With the administration of ordinary therapeutic doses of allopurinol, the excretion of uric acid plus the precursoro oxypurines finally stabilized at close to 50 per cent. of the. 
original uric acid excretion in five of the fourteen patients. In the entire group, the deficit of urinary purine averaged 35 per cent. (Rundles and others, 1966b).

The possibility that this deficit resulted from the re-utilization of hypoxanthine and xanthine when these purines were protected from oxidative degradation was considered, as well as the possible suppression of de novo purine biosynthesis (Pomales, Bieber, Friedman, and Hitchings, 1963). To obtain some clue to the mechanism involved, the effect of feeding exogenous purine was studied in two patients with chronic granulocytic leukaemia in remission (Rundles and others, 1963, 1966b).

Both these patients were maintained on a constant protein, constant purine diet, and when equilibrium was reached, they were given $1.5 \mathrm{~g}$. inosine by mouth daily for 3 days. The inosine was selected to provide a readilyabsorbed source of hypoxanthine. During its administration, its effect on the excretion of uric acid and precursor oxypurines was studied. When inosine was given without allopurinol, the urate increment in the urine accounted for 73 per cent. of the purine administered. When it was given with allopurinol, the urinary purine increment was only 35 to 40 per cent. of the purine administered.

The total data which has now accumulated indicate that there is considerable re-utilization of hypoxanthine and xanthine when these oxypurines are protected from the action of xanthine oxidase (Pomales and others, 1963). The amount of reutilization appears to be great enough to account for the deficit in purine excretion, although the accumulation of normal nucleotides may control purine biosynthesis to some extent by normal feedback inhibition (Rundles and others, 1966a).

As reported earlier today, studies of the metabolic fate of allopurinol showed that this compound was quickly absorbed from the gastrointestinal tract, to a total extent of at least 80 per cent., and that within a period of about 6 hours it was converted in large part to 4, 6-dihydroxypyrazolo $(3,4-d)$ pyrimidine (DHPP, allo-xanthine). Some 5 per cent. or less of a given dose of allopurinol appeared in the urine during the same period and some was converted to an allopurinol riboside (Elion, Kovensky, Hitchings, Metz, and Rundles, 1966a). Although allopurinol was a much more potent inhibitor of xanthine oxidase in vitro than allo-xanthine, it was thought that the latter compound, accumulating in the blood and being retained at a more constant and elevated level, might contribute significantly to the net therapeutic effect. A preliminary study of the effect of giving the allopurinol metabolite, alloxanthine, to three representative patients was accordingly designed and carried out.
The first patient (E.B.W.) had chronic granulocytic leukaemia in remission maintained by small doses of L-phenylalanine mustard. She was given $450-600 \mathrm{mg}$. allo-xanthine daily for 3 days. The serum uric acid concentration dropped from $3 \cdot 8-4 \cdot 0$ to $3 \cdot 3 \mathrm{mg} . / 100 \mathrm{ml}$. and the urate excretion from 390 to $330 \mathrm{mg} . / 24 \mathrm{hrs}$. There was a slight concomitant rise in hypoxanthine and xanthine excretion. These changes were somewhat less than those produced by the same dose of allopurinol.

The other two patients who were given allo-xanthine had gout. Case J.T.S. had extensive tophaceous deposits and was partially disabled by hypertensive cardiovascular disease. He had developed chronic arthritis with joint deformity and tophi after having been known to have gout for only 3 years. He was given allopurinol initially for a period of 22 months and during this time his performance status improved considerably and his tophi became smaller and softer. A somewhat latent chronic dermatitis became much worse, however, and finally exfoliated. When the allopurinol administration was suspended, the skin condition improved, but it became worse again in a short period of time when the therapy was resumed. He was then maintained on a prednisone-colchicine-probenecid regimen for a few months, but finally began to have increasing joint stiffness and developed new tophi. A study of the effect of alloxanthine on his purine metabolism was then carried out. The compound was given in a dose of 600 to $800 \mathrm{mgo}$ daily. During the pre-trial period, the serum uric acig level was 8 to $10 \mathrm{mg} . / 100 \mathrm{ml}$, and the urinary urate excretion about $790 \mathrm{mg}$. per day. After taking $600 \mathrm{mg}$ allo-xanthine daily for 5 days, the serum uric acid lev had fallen to $6.25 \mathrm{mg} . / 100 \mathrm{ml}$. and the urinary urate excretion to $580 \mathrm{mg} . / 24$ hours. After he had taken $800 \mathrm{mg}$. of the compound daily for 14 days, the serum uric acid was $4.35 \mathrm{mg} . / 100 \mathrm{ml}$., and the urinary urate excretion $315 \mathrm{mg} . / 24$ hours. While he was receiving allo-xanthine he had no evidence of adverse cutaneous or other drug reaction.

E.A.B., a Negro woman 56 years of age, had had gout for 10 years which had responded poorly to the intermittent administration of a uricosuric agent and colchicine. Acute attacks of joint inflammation became increasingly frequent and finally generalized joint involvement disabled her almost completely for a period of 7 months. Examination at the end of this time showed that most of her peripheral joints were swollen and tender. She had a mild anaemia, persistent proteinuria, and azotaemia. $A$ 응 regimen using allopurinol and colchicine was begun with little expectation of achieving a good result, but within a 윽 few weeks the joint inflammation subsided and the performance status improved so that she was able to return $\frac{D}{2}$ to work. The blood urea nitrogen, which had been raised during the pre-treatment period to 38 to $42 \mathrm{mg} . / 100 \mathrm{~N}$ $\mathrm{ml}$., fell to $21 \mathrm{mg} . / 100 \mathrm{ml}$. in a few weeks and then remained within normal limits. After she had taken 0 allopurinol for 7 months, however, she developed a skin $\mathbb{W}$ rash with depigmentation which became increasingly severe. She finally developed complete alopecia, at which time allopurinol therapy was suspended. During $\frac{}{\Phi}$ the following weeks her skin improved and her hair $\mathscr{D}$ began to grow again, slowly. In a month or two, how- 
ever, hyperuricaemia reappeared, along with the recurrence of acute and chronic arthritic manifestations. She was then treated with prednisone and colchicine, but despite this gradually became incapacitated again. The serum uric acid level rose to 13 to $15 \mathrm{mg} . / 100 \mathrm{ml}$., the urinary urate excretion to $300 \mathrm{mg} . / 24 \mathrm{hrs}$, and the blood urea nitrogen to about 49 to $65 \mathrm{mg} . / \mathrm{ml}$.

A study of the metabolic effects of allo-xanthine was then undertaken. She was given $300 \mathrm{mg}$. daily for 3 days without side-effects, and then the dose was increased to $600 \mathrm{mg}$. daily, which was tolerated equally well. After 10 days of therapy, the serum uric acid level had dropped to $5.6 \mathrm{mg} . / \mathrm{ml}$., the urinary urate excretion to $200 \mathrm{mg}$. $/ 24$ $\mathrm{hrs}$, and the blood urea nitrogen to about $30 \mathrm{mg} . / 100 \mathrm{ml}$. The arthritic symptoms subsided, and she became normally active again.

A few weeks later, allo-xanthine therapy was temporarily suspended. Within a few days, joint pain and swelling with localized heat developed, the serum uric acid level rose to $15 \mathrm{mg} . / 100 \mathrm{ml}$. and the blood urea nitrogen to $51 \mathrm{mg}$. $/ 100 \mathrm{ml}$. The administration of alloxanthine was resumed, using the suboptimal dose of
$300 \mathrm{mg}$. daily, and over a period of several weeks it controlled fairly satisfactorily most of her clinical and $\frac{0}{\omega}$. chemical abnormalities, with no evidence of adverse side-effects.

In reference to the long-term therapeutic use of $\frac{}{5}$ allopurinol which would be required for the control $\frac{}{\omega}$ of gout, chronic hyperuricaemia, etc., the compound $\vec{\nabla}$ does not appear to affect the intrinsic xanthine oxidase activity levels of the recipients. Maintenance doses produced a fairly constant therapeutic effect $\vec{O}$ for as long as 2 or 3 years, and, in some instances, the maintenance dose requirement seemed to drop some- $\vec{\omega}$ what. One patient who had been taking allopurinol for several months was found to convert allopurinol $\frac{0}{\alpha}$ to allo-xanthine at a rate considerably slower than N some who were given single doses of the drug (Elionis and others, 1966a). At the moment it appears thato the synthesis of xanthine oxidase does not seem to be induced by the abnormal substrate nor depressed by the prolonged presence of the inhibitor. 